\title{
E93 expression and links to the juvenile hormone in hemipteran mealybugs with insights on
}

\section{female neoteny}

Isabelle Mifom Vea ${ }^{1,2}$, Sayumi Tanaka ${ }^{2}$, Tomohiro Tsuji ${ }^{2}$, Takahiro Shiotsuki ${ }^{3,4}$, Akiya Jouraku ${ }^{3}$, and Chieka Minakuchi ${ }^{2}$

1 University of Edinburgh, Institute of Evolutionary Biology, Edinburgh, UK

2 Nagoya University, Graduate School of Bioagricultural Sciences, Nagoya, Japan

3 National Agriculture and Food Research Organization, Tsukuba, Japan

4 Shiname University, Faculty of Life and Environmental Science, Matsue, Japan

Corresponding author: Isabelle M. Vea (isabelle.vea@gmail.com)

\section{Abstract}

Insect metamorphosis generates reproductive adults and is commonly accompanied by the direct or indirect development of wings. In some winged insects, the imago is altered by life history changes. For instance, in scale insects and mealybugs, reproductive females retain juvenile features and are wingless. The transcription factor E93 triggers metamorphosis and plays in concert with the juvenile hormone pathway to guarantee the successful transition from juvenile to adult. We previously provided evidence of an atypical down-regulation of the juvenile hormone pathway during female adult development in the Japanese mealybug. Here, we further investigate how E93 is involved in the production of neotenic wingless females, by identifying its isoforms, assessing their expression patterns and evaluating the effect of exogenous juvenile hormone mimic treatment on E93. This study identifies three E93 isoforms on the 5' end based on Japanese mealybug cDNA and shows that female development occurs with the near absence of E93 transcripts, as opposed to male metamorphosis. Additionally, while male development is typically affected by exogenous juvenile hormone mimic treatments, females seem to remain insensitive to the treatment, and upregulation of the juvenile hormone signaling is not observed. Furthermore, juvenile hormone mimic treatment on female nymphs did not have obvious effect on E93 transcription, while treatment on male prepupae resulted in decreased E93 transcripts. In this study, we emphasize the importance of 


\section{Graphical abstract}

Extreme sexual dimorphism in hemimetabolous mealybugs

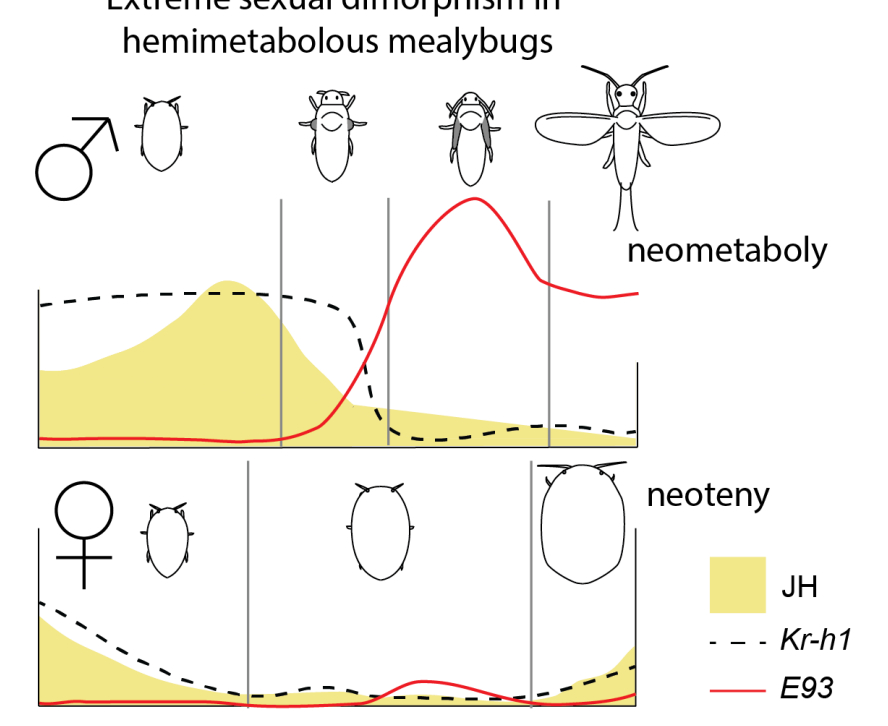

Highlights expression. transcription. males and other insects.

examining cases of atypical metamorphosis as complementary systems to provide a better

understanding on the molecular mechanisms underlying insect metamorphosis. For instance, the factors regulating the expression of $E 93$ are largely unclear. Investigating the regulatory mechanism of E93 transcription could provide clues towards identifying the factors that induce or suppress E93 transcription, in turn triggering male adult development or female neoteny.

Link between juvenile hormone and E93

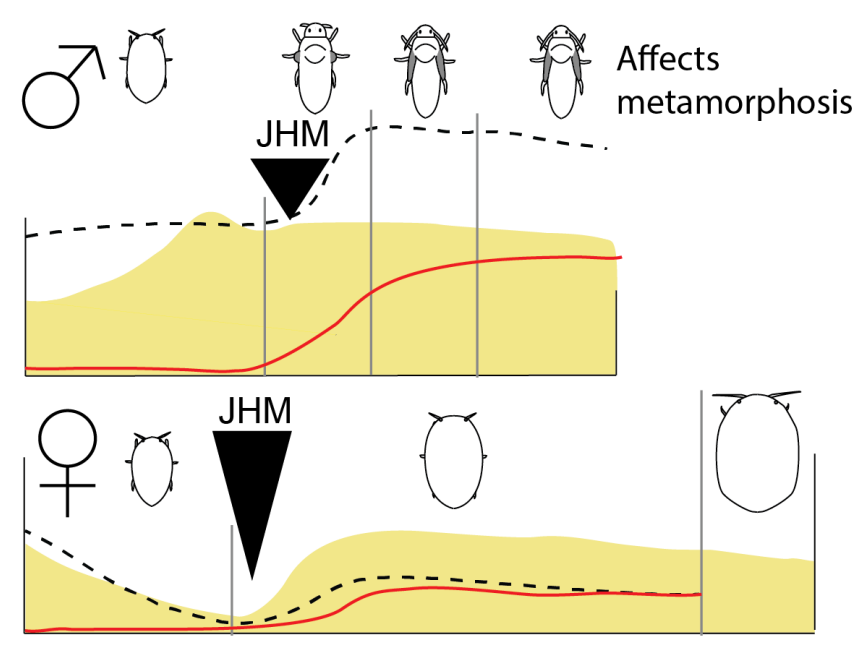

Prolonged last nymphal instar but low sensitivity to $\mathrm{JH}$

- Neotenic female Planococcus kraunhiae (Japanese mealybug) develops with low E93

- E93 expression pattern during male development is typical to other insects.

- Juvenile hormone mimic treatment on male prepupae results in decreased E93 transcripts.

- Juvenile hormone mimic treatment on female nymphs does not have obvious effects on E93

- Female mealybugs have low sensitivity to juvenile hormone mimic treatments compared to 


\section{Introduction}

The evolution of metamorphosis undeniably contributed to the diversity of insect forms, life

histories and increasing opportunities for ecological niche exploitation (Grimaldi and Engel, 2005;

Truman and Riddiford, 1999). While two types of metamorphosis -- holometaboly and

51 hemimetaboly - predominate in insects, in a few lineages, life cycles deviate to form unusual

52 developmental instances, nonetheless leading to important adaptations. For example,

hypermetamorphosis, manifested by two types of larvae, arose from holometaboly. Found in

Strepsiptera, Meloidae and Rhipiphoridae beetles, neuropteran Mantispidae and many Hymenoptera

and some Diptera, hypermetamorphosis is often associated to parasitic life (Truman and Riddiford,

2002) or predatory habits (Belles, 2011). Neometaboly is another metamorphosis found in plant

sap-feeding hemimetabolous Paraneoptera (Aleyrodoidea, Thysanoptera, and male Coccomorpha),

in which the formation of quiescent stages (prepupae and pupae) is reminiscent to holometaboly

(Belles, 2011).

Two hormones orchestrate insect metamorphosis: ecdysone and the juvenile hormone (JH).

$\mathrm{JH}$, in particular, dictates the identity of subsequent stages and is therefore of special interest for

understanding how peculiar life cycles arise. An essential player, the transcription factor E93,

triggers adult metamorphosis and is universally up-regulated at the end of insect juvenile cell death process in the prepupa (Baehrecke and Thummel, 1995; Buszczak et al., 2000; Lee et al., 2000) and acts as a developmental switch to control the responsiveness of target genes during metamorphosis (Mou et al., 2012). Later, functional studies on E93 orthologs in both hemimetabolous and holometabolous species confirmed E93 is a universal adult specifier in insect metamorphosis (Ureña et al., 2014). Finally, a communication between E93 and JH signaling pathway exists. In fact, Krüppel homolog $1(K r-h 1)$, an early response gene of JH signaling, acts as

71 a repressor of $E 93$ until the onset of adult metamorphosis: knocking down $K r-h 1$ at the penultimate 
juvenile instar of hemimetabolous Blattella germanica results in the early increase of E93 (Belles and Santos, 2014). Thereafter, functional studies in hemimetabolous Cimex lectularius (Gujar and Palli, 2016) and Tribolium castaneum pupal stage (Ureña et al., 2016) confirmed the interaction of JH signaling and E93 in other insect lineages. Finally, the direct transcriptional repressor role of $\mathrm{Kr}$ h1 on E93 promoter region was confirmed in Bombyx mori (Kayukawa et al., 2017). features: neoteny. Considered merely as curiosities, neotenic forms originated multiple times in various insects and can be associated to particular adaptive traits. Examples include parasitism in al., 2007; South et al., 2011). Nevertheless, few studies have addressed the underlying molecular mechanisms of neoteny. The emergence of diverse ways to metamorphose could be associated to the change in maturation timing (heterochrony), which implies that variations in controlling hormones may be an essential factor in establishing these forms (Gould, 1977). As such, femalespecific neotenic forms should be tightly linked to the reproductive function of JH. So far, the main hypothesis for the creation of juvenile-like reproductive females resides in excessive levels of $\mathrm{JH}$, simultaneously affecting female developmental progress and the timing of activating reproductive function (Matsuda, 1976).

Excessive JH titers are indeed observed in termite neotenics of Reticulitermes speratus. Here, the female reproductive neotenic caste shows significantly higher JH titers than those of the nymphs or worker castes. Additionally, knocking down JH receptor (RsMet) depletes vitellogenin transcript levels. However, it is still unclear whether the phenotypic features attributed to neotenics are affected (Saiki et al., 2015). The only other molecular studies on insect neotenic forms were undertaken on holometabolous insects, where the role of ecdysone was investigated as the responsible factor. Strepsiptera (twisted-wing insects) display sex-specific neotenic forms, whereby 
specifier broad in Xenos vesparum was also examined. In holometabolous insects, broad is upregulated during the last larval instar, at the onset of metamorphosis with an ecdysone titer increase (Kiss et al., 1988; Konopova and Jindra, 2008; Parthasarathy et al., 2008; Uhlirova et al., 2003). In $X$. vesparum, only males that undergo metamorphosis showed the increase of broad BTB domain expression, while it was not observed in last larval instar females (Erezyilmaz et al., 2014). Finally,

103 Cecidomyiidae (gall midges) possess a facultative paedogenetic life cycle where ovaries then differentiate and grow precociously in the larval stage. In this instance, a shift in timing of ecdysone receptor and ultraspiracle expression kick-starts the facultative life cycle and creates larval reproductive females (Hodin and Riddiford, 2000).

Scale insects and mealybugs (Coccomorpha) belong to Hemiptera, an insect order that mostly nymphal instars with progressive wing growth. Males undergo two quiescent stages reminiscent to

111 females retain juvenile features, as they develop through successive molts without wing growth, reduction of nymphal stages and features linked to mobility in many species (Gullan and Kosztarab, 1997). This life history trait not only gives rise to extremely sexually dimorphic organisms, but 114 offers a successful strategy, as plant-sap feeding insects, to allocate energy to reproduction, 115 sedentarize and adapt to their host-plant habitats. Female scale insects and mealybugs are often 116 described as neotenic (Danzig, 1980; Koteja, 1990). However, which type of neoteny, or the 117 mechanisms by which adult females keep juvenile features remains unknown. This prevents us 118 from understanding the link between the development and evolution of neoteny. More importantly, 119 this lineage includes some of the most damaging agricultural pests in human activities, likely a 120 consequence of the evolution of neotenic females and their adaptive life history to host plants. 
124 addition to significant differences observed in JH early-response gene $K r-h 1$ when male and female

125 mealybugs start to differentiate, we reported that JH signaling remained unusually low throughout

126 female adult development, suggesting a different $\mathrm{JH}$ regulation of female neoteny in this case (Vea

127 et al., 2016). To further examine the involvement of E93 in female neoteny, in relation to the $\mathrm{JH}$

128 signaling, we compared the expression pattern of E93, in P. kraunhiae male and female

129 postembryonic development and performed hormonal assays using a JH mimic, pyriproxyfen. We

130 hypothesize that in scale insects, females fail to express E93, resulting in the maintenance of

131 juvenile features in their external morphology even after reproductive maturation. Additionally, we

132 test whether increasing levels of $\mathrm{JH}$ during the last nymphal instars in females readjusts the

133 expression of $K r-h 1$ at similar levels as seen in males, which in turn could allow to initiate $E 93$

134 expression. It turned out that females are insensitive to exogenous JHM treatment in the context of 135 the effects on $K r-h 1$ and E93 expression as well as on adult development. 


\section{Materials and methods}

\subsection{Mealybug rearing and sampling strategy}

139 Mealybug culture and sampling strategy for gene expression profile are described in a previous

study on JH variations in the P. kraunhiae (Vea et al., 2016). In this study, we carried out an

141 independent sampling to ensure reproducibility of the previous study. As such, we collected

142 samples every 24 hours after oviposition. Eggs oviposited during the first day were used for male-

143 biased samples and eggs oviposited during the fifth day for female-biased samples (see Vea et al.,

1442016 for sex-biased sample strategy). All stages are abbreviated as follows: $\mathrm{E}=$ embryonic stage

145 after oviposition, N1= first-instar nymph, N2= second-instar nymph, N2f= female second-instar

146 nymph, $\mathrm{N} 2 \mathrm{~m}=$ male second-instar nymph, N3 = female third-instar nymph, pre=male prepupa, $\mathrm{pu}=$

147 male pupa, $\mathrm{m}=$ male adult, $\mathrm{f}=$ female adult.

\section{2. cDNA cloning and identification of sequences}

The total RNA of pooled individuals from different stages was extracted with TRIzol (as described

in Vea et al., 2016) and Oligo-dT-primed reverse transcription was performed with the PrimeScript

II $1^{\text {st }}$ strand cDNA synthesis kit (Takara Bio, Shiga, Japan). The conserved region of E93 sequence in B. germanica was blasted against a transcriptome of $P$. kraunhiae [accession number

DRA004114; (Sugahara et al., 2015)], and primers for RT-PCR were designed to amplify a partial region of the gene. Primers for RACE PCR were designed based on this partial sequence, and 5' and 3' RACE was conducted with SMARTer RACE cDNA Amplification Kit (Takara Bio USA, Inc., Mountain View, CA) in order to retrieve the full-length cDNA sequences. All PCR products were cloned in a pGEM-T Easy Vector (Promega, Madison, WI) and sequenced. DNA sequence

159 data were deposited in the DDBJ/EMBL-Bank/GenBank International Nucleotide Sequence 160 Database with the following accession numbers: PkE93 isoform 1 (LC374380), PkE93 isoform 2 
163 Total RNA was extracted from all samples using the sex-biased sampling as described in Vea et al.

164 (2016). Each sample consisted of 0.5 to $2 \mathrm{mg}$ of pooled individuals homogenized in TRIzol reagent, total RNA was extracted using nuclease-free glycogen (Thermo Fisher Scientific) as a carrier, and reverse transcribed using the PrimeScript RT reagent Kit with the gDNA Eraser (Takara Bio). Expression profiles for the post-oviposition development of males and females were established by quantifying the levels of transcripts for targeted fragments using absolute quantitative RT-PCR described previously (Vea et al., 2016). Six serial dilutions of a plasmid containing a fragment of each gene was used as the standard. Primer sequences for each PkE93 isoform used for qRT-PCR are listed in Table S1. The values obtained by the second derivative maximum (SDM) method were normalised with the ribosomal protein L32 (rpL32) transcript levels. Primers for PkrpL32, our reference gene, were from our previous study (Vea et al., 2016).

For JH mimic (JHM) treatments, we applied $2 \mu \mathrm{L}$ of pyriproxyfen ( $5 \mathrm{mM}$ dissolved in methanol) to batches of 3 to 5 male prepupae on a filter paper, 24-48 hours after molting. Excess chemical solution was immediately absorbed by the filter paper. After treatment, we waited for the solvent to evaporate completely before transferring them in $1.5 \mathrm{~mL}$ microcentrifuge tubes (Ina Optica, Osaka, Japan), bearing a paper disc ( $8 \mathrm{~mm}$ in diameter, $1.5 \mathrm{~mm}$ in thickness; Toyo Roshi, Japan) with 10 $\mu \mathrm{L}$ of distilled water to control humidity. Female N3D0 (0-24 h after molting to the N3 stage) were treated in the same manner except that $0.5 \mu \mathrm{L}$ of pyriproxyfen $(20 \mathrm{mM}$ dissolved in methanol) was applied on the tergite of individual N3D0. After the N3D0 started to move again, it was transferred into a glass dish containing a sprouted broad bean on top of a filter paper. The glass dishes were sealed with parafilm. Treated samples were left to incubate at $23^{\circ} \mathrm{C}$ for various numbers of days after treatment before being homogenized in TRIzol and stored at $-80^{\circ} \mathrm{C}$ for RNA extraction. RNA 


\subsection{Graphs and statistical analyses}

190 Graphs from qRT-PCR SDM values were generated using the R package ggplot2 and the statistical 191 significance of JHM effects were calculated by comparing the overall effect of the mimic over the 192 days using a linear model. We also tested the effect of JHM on individual days 4 and 8 after 193 treatment in females using the Student's $t$-test. All analyses using qRT-PCR SDM values are 194 detailed and publically available in GitHub and Zenodo

195 (https://zenodo.org/badge/latestdoi/116843862).

\section{Results and Discussion}

\subsection{Structure of E93 in P. kraunhiae}

200 characteristic of E93 (Siegmund and Lehmann, 2002) and highly conserved (Fig. 1A). Using 5' and 201 3' RACE PCR combined to designed primers from the conserved region, we cloned and sequenced 202 three complete transcripts of 5008, 5056 and $5233 \mathrm{bp}$ long. All of them resulted from either usage 203 of different transcription initiation site and/or alternative splicing on 5'end only (Fig. 1B). Each 204 isoform was arbitrarily designated as PkE93-1, PkE93-2 and PkE93-3. The region common to all 205 transcripts counts $4676 \mathrm{bp}, P k E 93-1$ and $P k E 93-3$ predicted protein sequence is of 1050 aa, while 206 PkE93-2 has a predicted protein sequence of 1090 aa. In summary, $P k E 93-1$ and $P k E 93-3$ differ in 207 the 5' untranslated region, while PkE93-2 has a longer coding region.

\subsection{Sex-specific expression profiles of E93}

We first examined the expression profile of each PkE93 isoform during the post-

211 oviposition development in male and female mealybugs, using absolute qRT-PCR (Fig. 2A).

212 PkE93-1 isoform has the highest expression compared to the two other isoforms. Generally, PkE93-

2131 stays at low levels in the embryo, N1 and N2 in both males and females (Fig. 2A; top). In males

214 only, PkE93-1 suddenly increases at the beginning of prepupa and reaches a peak of expression 
215 before adult metamorphosis. In females, however, PkE93-1 does not show such dramatic

216 expression, although slight increases at the end of N2 and N3 are observed (Fig. 2B). PkE93-2

217 isoform followed a similar expression pattern although around 20-fold lower (Fig. 2A; middle),

218 which suggests that $P k E 93-2$ may be a minor isoform. Finally, PkE93-3, despite its also lower

219 expression, shows a distinct pattern during the embryonic stage (Fig. 2A; bottom), with peaks of

220 expression in both males and females, similar to that of $\mathrm{PkKr}-\mathrm{hl}$ (Fig. S1). At the end of N2,

221 PkE93-3 expression also increases in males but decreases during the pupal stage, while its

222 expression reaches near-zero levels after N2 in females. PkE93 expression differs between males

223 and females more strikingly than the early JH-response gene $P k K r-h 1$. This is especially true

224 towards the end of post-embryonic development, where a peak of expression is observed in males,

225 but not in females. At this point, males enter quiescent stages (prepupa and pupa) where wings

226 develop. In contrast, females molt only once then become reproductively mature but retain juvenile

227 features. Along with previous results on $P k K r-h 1$ and $P k b r$ (Vea et al., 2016), we suggest that sex-

228 specific expression patterns of PkE93 may contribute to the development of sexual dimorphism in 229 mealybugs.

3.3. Expression pattern of male mealybugs consistent with JH signaling of other insects

The origin of holometaboly has been debated for decades. Although the consensus is that

233 endocrinological changes are responsible for the transition between hemimetaboly and

234 holometaboly, two hypotheses were advanced regarding the details of stage homologies [see details

235 in (Truman and Riddiford, 1999)]. More recently, based on study of E93 expression in $B$.

236 germanica, it was proposed that the last nymphal stage in hemimetabolous insects is

237 ontogenetically homologous to the pupal stage of holometabolous insects because of the similar

238 E93 expression pattern during these stages (Belles and Santos, 2014). Furthermore, the propupal

239 and pupal stages in neometabolous Thysanoptera, could be together homologous to the

240 holometabolous pupal stage (Minakuchi et al., 2011). In scale insects, a similar neometabolous 
241 development occurs in males (stages traditionally coined as "prepupa" and "pupa") and our

242 expression profile pattern in male P. kraunhiae (Fig. 2A) shows that PkE93 expression starts at the

243 beginning of the prepupa and peaks at the beginning of the pupal stage. Finally, hormonal treatment

244 using JHM at the beginning of the prepupal stage, when $K r$ - $h 1$ expression drops suddenly, prevents

245 adult metamorphosis by creating a supernumerary pupal stage (Vea et al., 2016). In addition to

246 maintaining high levels of $P k K r-h 1$ for at least six days, JHM treatment results in the significant

247 decrease of $P k E 93-1$ and $P k E 93-2$, while $P k E 93-3$ is not significantly affected (Fig. 3A). Although

248 functional analyses are still necessary to confirm this in mealybugs, the effects of JHM on E93

249 expression in neometabolous males are congruent with previous results found in other

250 holometabolous and hemimetabolous insects, and $K r-h 1$ and $E 93$ must be acting as reciprocal

251 inhibitors.

\subsection{Female atypical regulation of JH signaling and E93}

Based on our expression profile of PkE93 in females, all isoforms are very low throughout

the successive molting events, contrasting with male neometabolous development. However, if we compare $P k E 93$ and $P k K r-h 1$ expression only during the last instar nymphs, we see that even at very low levels, a slight decrease of $P k K r-h 1$ is accompanied with a small peak of expression of PkE93-1 (Fig. 2B). Although the female expression is 20-fold lower than in males, this small peak could explain the sexual maturation of females after N3, while somatic differentiation does not occur in the imago. Additionally, this small peak likely indicates tissue-specific expression, like ovaries and related reproductive organs. As $P k K r-h 1$ expression begins to decrease progressively at the penultimate nymphal stage in females (Figs. 2B and S1; Vea et al. 2016), we decided to increase the expression of $K r$ - $h l$ with JHM treatment at the early last nymphal stage (N3D0). By doing so, we assessed whether $K r-h 1$ can artificially reach a threshold necessary for the switch to adult fate controlled by E93. Twenty $\mathrm{mM}$ of pyriproxyfen were applied on N3D0, concentration 
individuals died before the last molt (Table 1). The 12 survivors (63\%), showed a tendency to

268 prolong the $\mathrm{N} 3$ stage. While the control samples molted $9.6 \pm 1.5$ days $(\mathrm{N}=14)$ after treatment, 12 out

269 of $19 \mathrm{JHM}$-treated individuals molted to adult stage $13 \pm 2.5$ days after treatment. We also followed

270 the effect of JHM treatment on PkKr-h1 and PkE93 expression (Fig. 3B), one to eight days after

271 treatment (last days before the female adult molt). At $20 \mathrm{mM}$, pyriproxyfen induced the up-

272 regulation of $P k K r-h 1$ after four days, which then lasted several days. However, it is worth

273 mentioning that $P k K r-h 1$ levels were significantly lower than the response observed in males, even

274 with a concentration of pyriproxyfen four times lower. We suggest that female mealybugs may have

275 mechanisms preventing them to respond to JH as sensitively or efficiently as in males. Previously,

276 we showed that PkMet and PkTai, forming the JH receptor complex, were highly expressed at the

277 end of male development, while in females the expression remained low (Vea et al., 2016). After

278 JHM treatment on females at N3D0, although $P k K r-h 1$ starts to be affected only four days after

279 treatment by an upregulation, the expression of all PkE93 isoforms does not change significantly

280 over time (interaction between treatment and time not significant; Fig. 3B). However, when

281 removing the interaction, the treatment alone leads to an overall significant effect for two isoforms;

282 this is probably explained by the increase in expression at Day 2, Day 4 and Day 8 after treatment

283 for $P k E 93-1$ and $P k E 93-2$. We therefore conclude that applying JHM on early ultimate juvenile

284 instars in females does not significantly change $P k E 93$ expression overtime, but a small effect

285 might be observed at Day 8 after treatment by an increase of PkE93-1 and PkE93-2.

Several studies have demonstrated that at the onset of metamorphosis in holometabolous insects, juvenile instars need to reach a critical weight for adult metamorphosis (Davidowitz et al., 288 2003; Nijhout, 2003; Nijhout et al., 2014). In mealybugs, males and females are phenotypically 289 identical before their development starts to diverge. If we consider that critical weight is a factor in 290 at least male metamorphosis, the threshold should be reached at the start of prepupa, where we see

291 the sudden decrease of $P k K r-h 1$. By contrast, females should attain their critical weight for 292 metamorphosis in N3 stage. In females, $P k K r$ - $h 1$ down-regulation takes place in a progressive 
decrease from the middle of N2 rather than the sudden decline of transcripts. Moreover, Pkjhamt

294 (juvenile hormone acid O-methyltransferase), involved in the last steps of JH biosynthesis, starts

295 decreasing even at the beginning of N2, while in males, the transcripts are still expressed until

296 prepupa (Vea et al., 2016). This indicates an early arrest in JH synthesis in female development.

297 Another possibility is that a sudden decrease combined with attaining a threshold of $K r-h 1$

298 expression is necessary to induce E93 expression. In this case, maintaining low levels of $P k K r-h 1$

299 during female development may explain why PkE93 expression never peaks.

\section{Concluding remarks}

$\mathrm{JH}$ anti-metamorphic role in insect metamorphosis suggests that high levels of this hormone delays metamorphosis. Female neoteny has therefore been believed to be the result of disrupted JH downregulation leading to constantly high JH titers. Although this hypothesis was first proposed based only on the differential size of scale insect corpora allata (Matsuda, 1976), we recently reported contradictory evidence that the last juvenile stages in female Japanese mealybug develop under surprisingly lower JH titers compared with males (Vea et al., 2016). In this study, we provide additional data to support an atypical hormonal regulation in mealybugs, and show the first example of possible failure in E93 induction, linked to neotenic reproductive females in an hemimetabolous insect. The response of female development to $\mathrm{JH}$ modulations is intriguing and suggests that mealybug neotenic forms are insensitive to $\mathrm{JH}$ signaling as opposed to males, has consequences on E93 expression, and leads to extreme sexual dimorphism. So far, gene expression manipulation on mealybug juvenile stages by dsRNA injection has been ineffective. Alternatively, identifying suppressors of E93 promoter in neotenic females, coupled with functional studies through genome editing should provide novel insights on the function and interaction between E93 and JH signaling pathway in neotenic female scale insects. 
bioRxiv preprint doi: https://doi.org/10.1101/283556; this version posted March 16, 2018. The copyright holder for this preprint (which was not certified by peer review) is the author/funder, who has granted bioRxiv a license to display the preprint in perpetuity. It is made available under aCC-BY-NC-ND 4.0 International license.

\section{Acknowledgements}

320 We thank Jun Tabata for providing the Japanese mealybugs, Ken Miura and Xavier Belles for the

valuable comments on this study. All the work presented here was conducted at Nagoya University

and was funded by a JSPS postdoctoral fellowship for overseas researchers to IMV and a Grant-in-

aid for Scientific Research (15K07791) to CM from JSPS.

\section{References}

326

Baehrecke, E.H., Thummel, C.S., 1995. The Drosophila E93 gene from the 93F early puff displays stage- and tissue-specific regulation by 20-hydroxyecdysone. Dev. Biol. 171, 85-97. doi:10.1006/dbio.1995.1262

Belles, X., 2011. Origin and evolution of insect metamorphosis, in: Encyclopedia of Life Sciences ELS. John Wiley \& Sons, Ltd, Chichester, UK. doi:10.1002/9780470015902.a0022854

Belles, X., Santos, C.G., 2014. The MEKRE93 (Methoprene tolerant-Krüppel homolog 1-E93) pathway in the regulation of insect metamorphosis, and the homology of the pupal stage. Insect Biochem. Mol. Biol. 52, 60-68. doi:10.1016/j.ibmb.2014.06.009

Bocakova, M., Bocak, L., Hunt, T., Teraväinen, M., Vogler, A.P., 2007. Molecular phylogenetics of Elateriformia (Coleoptera): evolution of bioluminescence and neoteny. Cladistics 23, 477-496. doi:10.1111/j.1096-0031.2007.00164.x

Buszczak, M., Biology, W.S.C., 2000, 2000. Insect metamorphosis: out with the old, in with the new. Curr. Bio. 10, R830-R833. doi:10.1016/S0960-9822(00)00792-2

Danzig, E.M., 1980. Coccids of the far eastern USSR (Homoptera, Coccinea) with phylogenetic analysis of coccids in the world fauna, Nauka. ed. Leningrad.

Davidowitz, G., D'Amico, L.J., Nijhout, H.F., 2003. Critical weight in the development of insect body size. Evolution \& Development 5, 188-197. doi:10.1046/j.1525-142X.2003.03026.X

Erezyilmaz, D.F., Hayward, A., Huang, Y., Paps, J., Acs, Z., Delgado, J.A., Collantes, F., Kathirithamby, J., 2014. Expression of the pupal determinant broad during metamorphic and neotenic development of the strepsipteran Xenos vesparum Rossi. PLoS ONE 9, e93614. doi:10.1371/journal.pone.0093614

Gould, S.J., 1977. Ontogeny and phylogeny. Harvard University Press.

Grimaldi, D., Engel, M.S., 2005. Evolution of the Insects, 1st ed. Cambridge University Press, New York.

Gujar, H., Palli, S.R., 2016. Krüppel homolog 1 and E93 mediate juvenile hormone regulation of metamorphosis in the common bed bug, Cimex lectularius. Sci. Rep. 1-10. doi:10.1038/srep26092

Gullan, P.J., Kosztarab, M., 1997. Adaptations in scale insects. Annu. Rev. Entomol. 42, 23-50. doi:10.1146/annurev.ento.42.1.23

Higashi, M., Abe, T., 1997. Global diversification of termites driven by the evolution of symbiosis and sociality, in: Biodiversity. Springer, New York, NY, New York, NY, pp. 83-112. doi:10.1007/978-1-4612-1906-4_7

Hodin, J., Riddiford, L.M., 2000. Parallel alterations in the timing of ovarian ecdysone receptor and ultraspiracle expression characterize the independent evolution of larval reproduction in two species of gall midges (Diptera: Cecidomyiidae). Dev Genes Evol 210, 358-372. doi:10.1007/s004270050324

Kathirithamby, J., 2009. Host-parasitoid associations in Strepsiptera. Annu. Rev. Entomol. 54, $227-$ 249. doi:10.1146/annurev.ento.54.110807.090525 
Kayukawa, T., Jouraku, A., Ito, Y., Shinoda, T., 2017. Molecular mechanism underlying juvenile hormone-mediated repression of precocious larval-adult metamorphosis. Proc. Natl. Acad. Sci. U.S.A. 201615423-10. doi:10.1073/pnas. 1615423114

Kiss, I., Beaton, A.H., Tardiff, J., Fristrom, D., Fristrom, J.W., 1988. Interactions and developmental effects of mutations in the Broad-Complex of Drosophila melanogaster. Genetics 118, 247-259.

Konopova, B., Jindra, M., 2008. Broad-Complex acts downstream of Met in juvenile hormone signaling to coordinate primitive holometabolan metamorphosis. Development 135, 559-568. doi:10.1242/dev.016097

Koteja, J., 1990. Life History, in: Armored Scale Insects, Their Biology, Natural Enemies, and Control, Vol. a. Elsevier, New York, NY.

Lee, C.Y., Wendel, D.P., Reid, P., Lam, G., Thummel, C.S., Baehrecke, E.H., 2000. E93 directs steroid-triggered programmed cell death in Drosophila. Mol. Cell 6, 433-443.

Matsuda, R., 1976. Morphology and evolution of the insect abdomen: with special reference to developmental patterns and their bearings upon systematics. Pergamon Press.

Minakuchi, C., Tanaka, M., Miura, K., Tanaka, T., 2011. Developmental profile and hormonal regulation of the transcription factors broad and Krüppel homolog 1 in hemimetabolous thrips. Insect Biochem. Mol. Biol. 41, 125-134. doi:10.1016/j.ibmb.2010.11.004

Mou, X., Duncan, D.M., the, E.B.P.O., 2012, 2012. Control of target gene specificity during metamorphosis by the steroid response gene E93. Proc. Natl. Acad. Sci. U.S.A. 109, 29492954. doi:10.1073/pnas.1117559109

Nijhout, H.F., 2003. The control of body size in insects. Dev. Biol. 261, 1-9. doi:10.1016/S00121606(03)00276-8

Nijhout, H.F., Riddiford, L.M., Mirth, C., Shingleton, A.W., Suzuki, Y., Callier, V., 2014. The developmental control of size in insects. Wiley Interdiscip Rev Dev Biol 3, 113-134. doi:10.1002/wdev.124

Parthasarathy, R., Tan, A., Bai, H., Palli, S.R., 2008. Transcription factor broad suppresses precocious development of adult structures during larval-pupal metamorphosis in the red flour beetle, Tribolium castaneum. Mech Dev 125, 299-313. doi:10.1016/j.mod.2007.11.001

Roisin, Y., 2000. Diversity and Evolution of Caste Patterns, in: Termites: Evolution, Sociality, Symbioses, Ecology. Springer, Dordrecht, Dordrecht, pp. 95-119. doi:10.1007/978-94-0173223-9 5

Saiki, R., Gotoh, H., Toga, K., Miura, T., Maekawa, K., 2015. High juvenile hormone titre and abdominal activation of $\mathrm{JH}$ signalling may induce reproduction of termite neotenics. Insect $\mathrm{Mol}$ Biol 24, 432-441. doi:10.1111/imb.12169

Siegmund, T., Lehmann, M., 2002. The Drosophila Pipsqueak protein defines a new family of helix-turn-helix DNA-binding proteins. Dev Genes Evol 212, 152-157. doi:10.1007/s00427002-0219-2

South, A., Stanger-Hall, K., Jeng, M.-L., Lewis, S.M., 2011. Correlated evolution of female neoteny and flightlessness with male spermatophore production in fireflies (Coleoptera: Lampyridae). Evolution 65, 1099-1113. doi:10.1111/j.1558-5646.2010.01199.x

Sugahara, R., Jouraku, A., Nakakura, T., Kusakabe, T., Yamamoto, T., Shinohara, Y., Miyoshi, H., Shiotsuki, T., 2015. Two adenine nucleotide translocase paralogues involved in cell proliferation and apermatogenesis in the silkworm Bombyx mori. PLoS ONE 10, e0119429. doi:10.1371/journal.pone.0119429.s005

Truman, J.W., Riddiford, L.M., 2002. Endocrine insights into the evolution of metamorphosis in insects. Annu. Rev. Entomol. 47, 467-500. doi:10.1146/annurev.ento.47.091201.145230

Truman, J.W., Riddiford, L.M., 1999. The origins of insect metamorphosis. Nature 401, 447-452. doi: $10.1038 / 46737$

Uhlirova, M., Foy, B.D., Beaty, B.J., Olson, K.E., Riddiford, L.M., Jindra, M., 2003. Use of Sindbis virus-mediated RNA interference to demonstrate a conserved role of Broad-Complex in insect 
metamorphosis. Proc. Natl. Acad. Sci. U.S.A. 100, 15607-15612. doi:10.1073/pnas.2136837100

Ureña, E., Chafino, S., Manjón, C., Franch-Marro, X., Martín, D., 2016. The occurrence of the holometabolous pupal stage requires the interaction between E93, Krüppel-Homolog 1 and Broad-Complex. PLoS Genet 12, e1006020. doi:10.1371/journal.pgen.1006020

Ureña, E., Manjón, C., Franch-Marro, X., Martín, D., 2014. Transcription factor E93 specifies adult metamorphosis in hemimetabolous and holometabolous insects. Proc. Natl. Acad. Sci. U.S.A. 111, 7024-7029. doi:10.1073/pnas.1401478111

Vea, I.M., Tanaka, S., Shiotsuki, T., Jouraku, A., Tanaka, T., Minakuchi, C., 2016. Differential juvenile hormone variations in scale insect extreme sexual dimorphism. PLoS ONE 11, e0149459. doi:10.1371/journal.pone.0149459 


\section{Figures}

A

Homo sapiens LCoR-2 RKKRGRYR QYNSEILEEAIS VVMSGKMSVISAAOSIY GIPHSTLEYKVK ERLG TL Caenorhabditis elegans MBR1 RPKRGQYRKYDKNALDEAVR SVRR GEMTVHRA GSFF GVPH STLEYKVKERNLMR Blattella germanica E93 RPKRGKYRNYDRDSLIEAVRAVQRGEMS VHRAGSHF GVPH STLEYKVKERHLMR Planococcus kraunhiae E93 RPKR GKYRNYDR D LVEAVR AVQRGEMS VHRA GSYY GVPH STLEYKVKERHL MR

Tribolium castaneum E93 RPKRGKYRNYDR DSLVEAVR AVORGEMS VHRAGSYY GVPH STLE YKVKERHLMR Drosophila melanogaster E93 RPKR GKYRNYDR DSLVEAVKAVQR GEMS VHRA GSYY GVPH STLE YKVK ERHL MR Bombyx mori E93 RPKRGKYRNYDR D SUVEAVKAVORGEMS VHRAGSYYGVPH STLEYKVKERHLMR

B
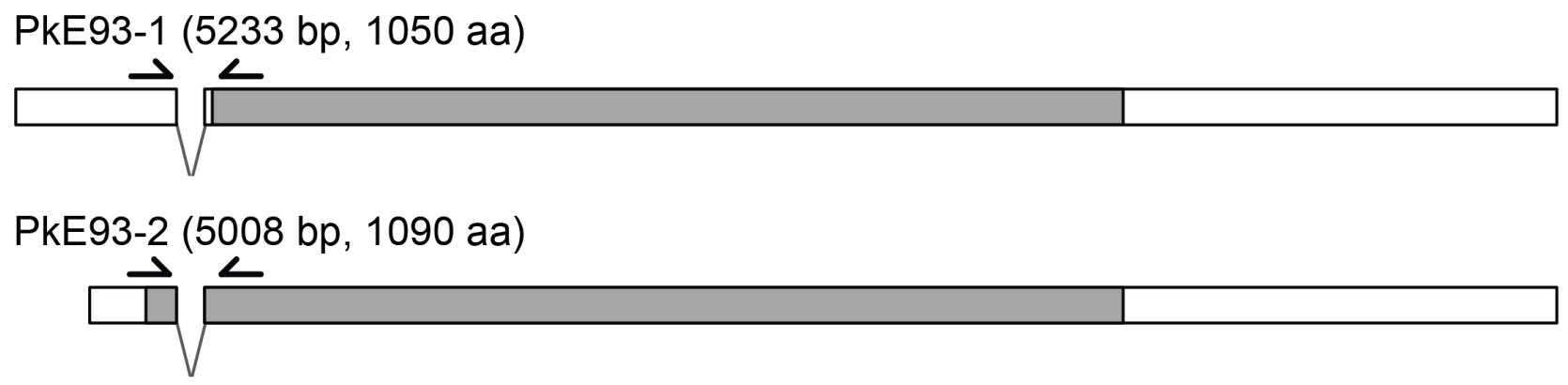

PkE93-3 (5056 bp, 1050 aa)

430 Figure 1: Identification of PkE93 sequence and the structure of its isoforms. A. Amino acid alignment of the Pipsqueak 431 DNA-binding domain with other insects' E93, Homo sapiens LCoR-2 and Caenorharbidtis elegans MBR1 [sequences 432 from (Ureña et al., 2014)]. B. General structure of cDNA sequences obtained from 5' and 3' RACE PCR. The sequences are identical on the 3' end, while the 5' end differ among the three isoforms, the common region is of 4676 bp starting from 3' end. Grey: open reading frame. Arrows: primers designed for qRT-PCR. Scale bar: 500 bp. 


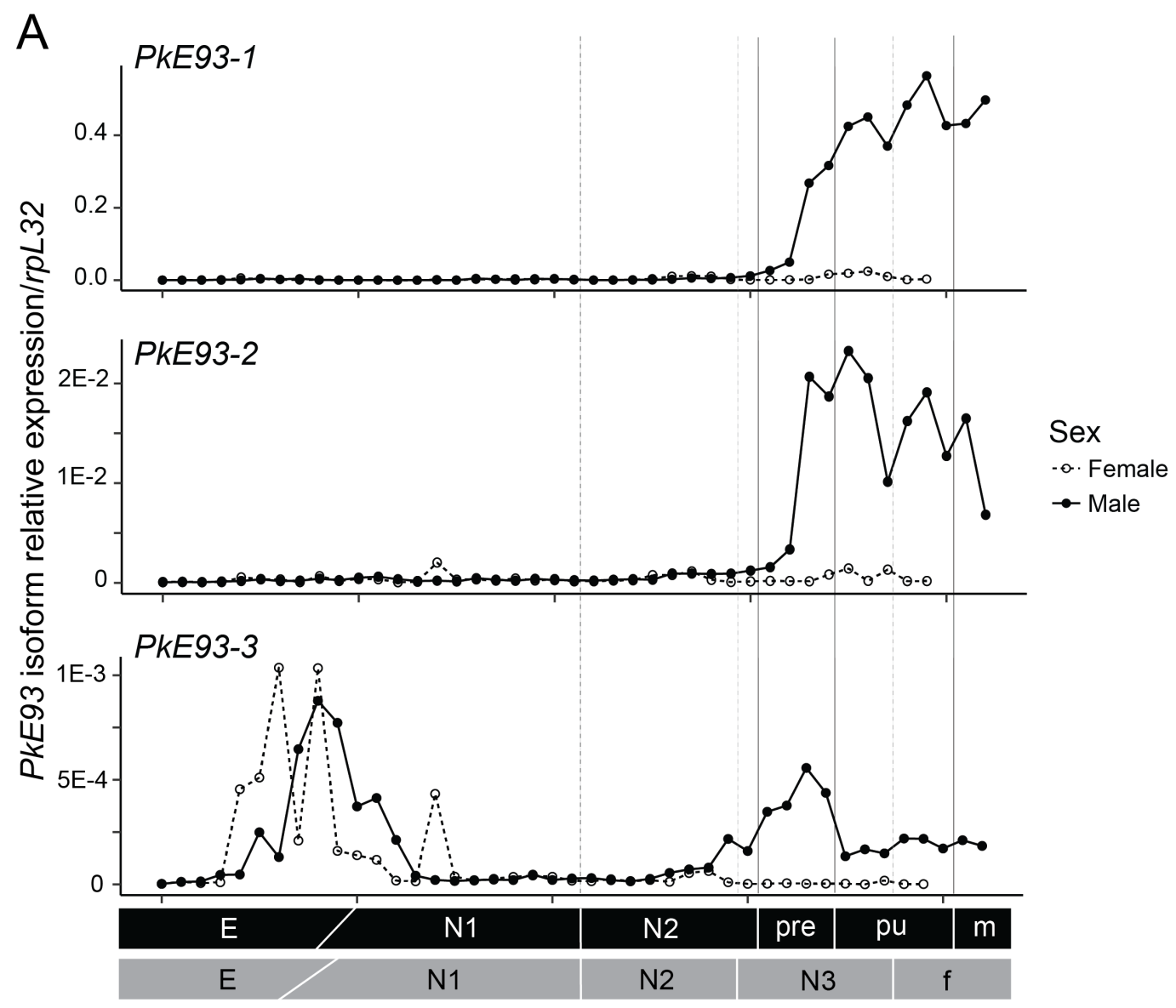

B

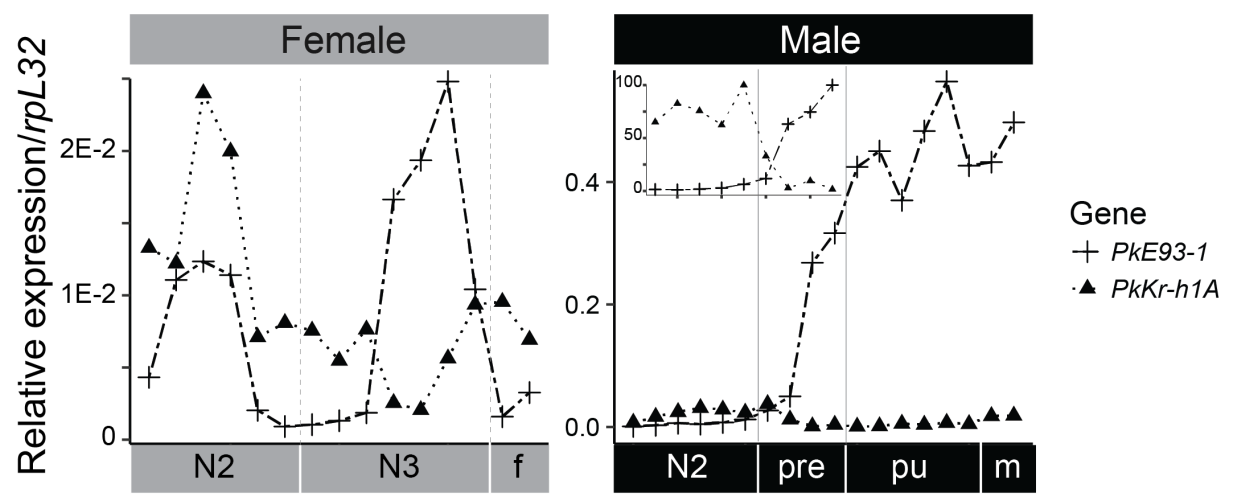

437 Figure 2: Expression profiles of $P k E 93$ isoforms throughout post-oviposition in males and females, and comparison 438 with those of $P k K r-h 1$ at the end of development. A. PkE93-1, PkE93-2 and PkE93-3 expression profiles from qRT439 PCR of samples collected every 24 hours from oviposition. The primers used for qRT-PCR are shown in Fig. 1 and 440 their sequences are listed in Table S1. B. Comparison of PkE93-1 and PkKr-h1A expression from the second-instar 441 nymph, when female and male can be differentiated, to the adult stage. The small graph on top left part of male 442 expression graph shows the relative expression in percentage when the switch of expression occurs between the two 443 genes. 

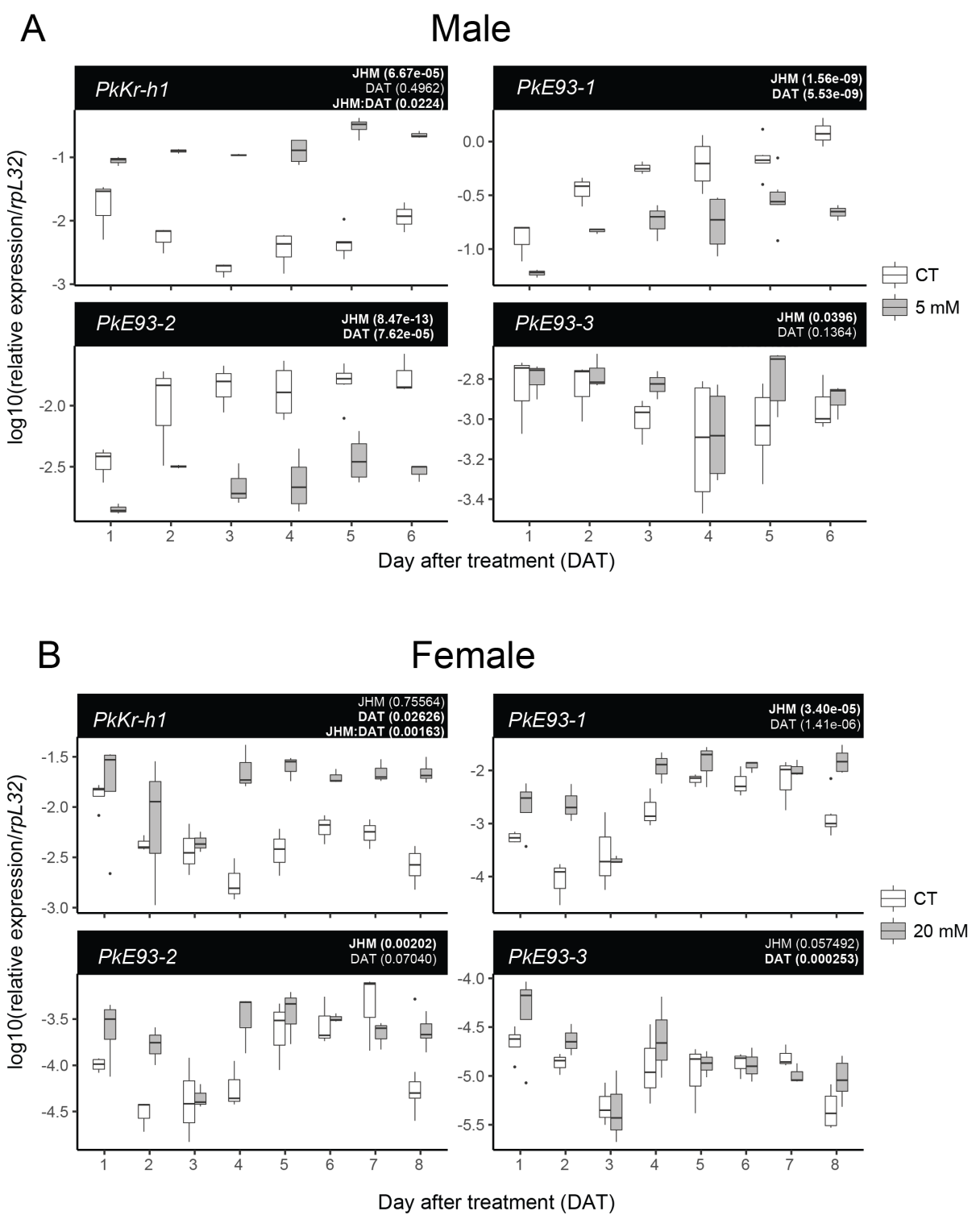

$445 \quad$ Figure 3: Effect of juvenile hormone mimic treatment on $K r-h 1$ and $E 93$ expression at the end of male and female

446 development. The transcript levels were analyzed with qRT-PCR, and normalized with the $r p L 32$ levels. A. Expression of PkKr-h1, PkE93-1, PkE93-2 and PkE93-3, 1 to 6 days after male 1 day old prepupae (PreD1) were treated with 5 mM pyriproxyfen or methanol. B. Expression of $P k K r-h 1, P k E 93-1, P k E 93-2$ and PkE93-3, 1 to 8 days after 0-24 h old third instar females (N3D0) were treated with $20 \mathrm{mM}$ pyriproxyfen or methanol. Statistical significance: we used a linear model in $\mathrm{R}$ on $\log 10$ transformed data, to test the significance of pyriproxyfen treatments and also considered day after treatment as a factor (see GitHub link in Materials and Methods for details on the analysis). P-values for each factor are indicated on top right side of each graph. We first considered an interaction between the two tested variables in our linear model. If the interaction is significant, the p-values of treatment (JHM), day after treatment (DAT) and interaction between them (JHM:DAT) are indicated (as it is the case for $P k K r-h l$ ). If no interaction was found, we used the linear model excluding the interaction and indicated p-values for treatment and day after treatment only (for all three PkE93 isoforms). We considered an effect significant when p-value $<0.01$.

\section{List of supplementary material}

Table S1: List of primers used for cloning, 5' and 3' RACE PCR and quantitative RT-PCR. 
460 Figure S1: Expression profile of $P k K r-h 1$ during male and female Japanese mealybug development 461 after oviposition

GitHub/Zenodo repository for data analysis: https://zenodo.org/badge/latestdoi/116843862 Protocol repository: protocols for sex biased collecting strategy in mealybugs

466 (dx.doi.org/10.17504/protocols.io.miac4ae) 
Table 1: Effect of pyriproxyfen treatment on last nymphal instar females on adult metamorphosis

\begin{tabular}{|c|c|c|c|c|c|c|c|c|c|c|c|c|}
\hline & $\mathrm{N}$ & $\begin{array}{c}\text { Number of } \\
\text { dead } \\
\text { individuals }\end{array}$ & $\begin{array}{l}\text { Number of } \\
\text { individuals } \\
\text { alive that } \\
\text { molted to } \\
\text { adult (\%) }\end{array}$ & & & & & & & & & \\
\hline & & (10) & 8 & 9 & 10 & 11 & 12 & 13 & 14 & 15 & 16 & $\begin{array}{l}\text { after } \\
\text { treatment) }\end{array}$ \\
\hline Methanol & 14 & $0(0)$ & $3(21)$ & $6(43)$ & $1(7.1)$ & $3(21)$ & $0(0)$ & $1(7.1)$ & $0(0)$ & $0(0)$ & $0(0)$ & \\
\hline $\begin{array}{c}20 \mathrm{mM} \\
\text { pyriproxyfen }\end{array}$ & 19 & $7(37)$ & $0(0)$ & $2(11)$ & $1(5.3)$ & $1(5.3)$ & $0(0)$ & $5(26)$ & $0(0)$ & $0(0)$ & $3(16)$ & \\
\hline
\end{tabular}

468

469

470

471

472 